\title{
Cambios en la capacidad física y algunas variables fisiológicas en pacientes con insuficiencia cardiaca a las 6 y 12 semanas de un programa de rehabilitación cardiaca
}

\author{
Changes in physical capacity and some physiological variables in patients with cardiac \\ insufficiency at 6 and 12 weeks of a cardiac rehabilitation program
}

\author{
Jhonatan Betancourt-Peña ${ }^{1,2 *}$, Óscar Ruiz-Serna ${ }^{3}$, Stephania Martinez-Gómez ${ }^{4}$,
} Paola Saavedra-Fernández ${ }^{5}$, Jorge K. Assis ${ }^{6}$ y Juan C. Ávila-Valencia ${ }^{1,7}$

${ }^{1}$ Facultad de Salud y Rehabilitación, Institución Universitaria Escuela Nacional del Deporte, Cali; ${ }^{2}$ Facultad de Salud, Escuela de Rehabilitación Humana, Universidad del Valle, Cali; ${ }^{3}$ Facultad de Salud, Fundación Universitaria María Cano, Medellín; ${ }^{4}$ Departamento UCI Neonatal, Clínica Nueva Cali, Cali; ${ }^{5}$ Servicio de Hospitalización y Urgencias, Clínica Nueva Cali, Cali; ${ }^{6}$ Departamento de Investigaciones Clínica de Occidente S.A., Cali; ${ }^{7}$ Facultad de Salud, Universidad Santiago de Cali, Cali. Colombia

\begin{abstract}
Resumen
Introducción: La insuficiencia cardiaca es un estado fisiopatológico y clínico en el cual el corazón es incapaz de aportar sangre de acuerdo con los requerimientos metabólicos periféricos, repercutiendo directamente sobre la capacidad aeróbica. La rehabilitación cardiaca durante 12 semanas tiene beneficios claros principalmente sobre la capacidad aeróbica, pero son pocos los estudios que detallan dichos cambios en el transcurso del proceso de intervención. Objetivo: Determinar los cambios en la capacidad aeróbica y algunas variables fisiológicas en pacientes con insuficiencia cardiaca, a las 6 y 12 semanas de su rehabilitación cardiaca. Método: Estudio de tipo descriptivo longitudinal. La muestra se constituyó por 23 pacientes vinculados a un programa de rehabilitación cardiaca, donde realizaron prueba de esfuerzo en tres momentos (inicio y semanas 6 y 12). Las variables cuantitativas se compararon en tres momentos utilizando la prueba de medidas repetidas hasta tres colas, teniendo en cuenta la homogeneidad y la igualdad de varianzas. La significación estadística fue de 0.05. Resultados: El consumo de oxígeno mostró un aumento de 3,6 $\mathrm{ml} / \mathrm{kg} / \mathrm{min}$ durante la rehabilitación, encontrando diferencias estadísticamente significativas en los tres momentos de medición, pero se evidenció un mayor incremento entre las semanas 1 y $6(p=0.00)$, cuando se registró un cambio de $2,2 \mathrm{ml} / \mathrm{kg} / \mathrm{min}$. La medición de la presión arterial sistólica/diastólica inicial y final mostró una disminución $(p<0.05)$. Conclusiones: En pacientes con insuficiencia cardiaca, el consumo de oxígeno obtuvo un incremento progresivo a lo largo de las 12 semanas de rehabilitación cardiaca, observándose su mayor incremento en las primeras 6 semanas de intervención.
\end{abstract}

Palabras clave: Falla cardiaca. Rehabilitación. Tolerancia al ejercicio. Ejercicio.

\section{Correspondencia:}

*Jhonatan Betancourt-Peña

E-mail: johnnatanbp @ hotmail.com
Fecha de recepción: 23-06-2019

Fecha de aceptación: 18-01-2021

DOI: 10.24875/RCCAR.M21000091
Disponible en internet: 29-11-2021 Rev Colomb Cardiol. 2021;28(5):502-509 www.rccardiologia.com 0120-5633 / C 2021 Sociedad Colombiana de Cardiología y Cirugía Cardiovascular. Publicado por Permanyer. Este es un artículo open access bajo la licencia CC BY-NC-ND (http://creativecommons.org/licenses/by-nc-nd/4.0/). 


\section{Abstract}

Introduction: Heart failure is a pathological and clinical state in which the heart is unable to provide blood according to peripheral metabolic requirements having a direct impact on aerobic capacity. Cardiac rehabilitation for 12 weeks has clear benefits mainly on aerobic capacity, but few studies detail such changes over the course of the intervention process. Objective: To determine changes in aerobic capacity and some physiological variables in patients with cardiac insufficiency, at 6 and 12 weeks after cardiac rehabilitation. Method: Longitudinal descriptive type study. The sample was made up of 23 patients linked to a cardiac rehabilitation program, where they performed stress testing at three times (start, weeks 6 and 12). Quantitative variables were compared in 3 moments using the repeated measurement test up to 3 tails, taking into account the homogeneity and equality of variances. The statistical significance was 0.05 . Results: Oxygen consumption showed an increase of $3.6 \mathrm{ml} / \mathrm{kg} / \mathrm{min}$ during rehabilitation, finding statistically significant differences in the three measurement moments but there was a greater increase between week 1 and $6(p=0.00)$ where a change of $2.2 \mathrm{ml} / \mathrm{kg} / \mathrm{min}$ was recorded. Measurement of initial and final systolic/diastolic blood pressure showed a decrease $(p<0.05)$. Conclusions: In patients with heart failure, oxygen consumption achieved a progressive increase over 12 weeks of cardiac rehabilitation, with its largest increase seen in the first 6 weeks of intervention.

Key words: Heart failure. Rehabilitation. Exercise tolerance. Exercise.

\section{Introducción}

Las enfermedades cardiovasculares causan $17.7 \mathrm{mi}-$ llones de muertes en el año, lo que las convierte en la primer causa de morbimortalidad en el mundo, y para el año 2020 los más afectados serán los países de medianos y bajos ingresos, como Colombia ${ }^{1}$. Por su parte, la insuficiencia cardiaca afecta aproximadamente a 40 millones de pacientes en todo el mundo²; en Latinoamérica se presenta en sujetos más jóvenes que en el resto del mundo y se relaciona con más frecuencia con cardiopatía isquémica. En Colombia existen cerca de 1.1 millones de pacientes con insuficiencia cardiaca, con una prevalencia estimada del $2.3 \%$ en la población general, y su tasa fue estimada en 5.54 por 100.000 habitantes en el año 2012'. A pesar de los avances en el diagnóstico y el tratamiento de la enfermedad, la prevalencia de insuficiencia cardiaca sigue en aumento, a menudo asociada con el daño del miocárdico, que de prolongarse comúnmente causa insuficiencia cardiaca ${ }^{3}$, lo que produce que el costo del cuidado de estos pacientes se incremente, en parte debido a las hospitalizaciones, ya que estas aumentan a medida que los tratamientos prolongan la supervivencia ${ }^{2}$.

Si bien la insuficiencia cardiaca se define como el estado fisiopatológico y clínico en el cual el corazón es incapaz de aportar sangre de acuerdo con los requerimientos metabólicos periféricos, una de sus características principales es el remodelamiento inadecuado del miocardio, lo que causa una disminución en la función de bomba del corazón, por lo cual la función ventricular de llenado y bombeo se ve comprometida funcionalmente. Es considerada el extremo final común de muchas de las enfermedades más prevalentes, como son la hipertensión arterial, la enfermedad coronaria, la diabetes mellitus y las valvulopatías, entre otras ${ }^{4}$.

Esta situación requiere un manejo farmacológico estricto, como también otras alternativas terapéuticas, tales como el ejercicio de predominio aeróbico ${ }^{5}$, con el fin de mejorar la disnea, la fatiga y la tolerancia al ejercicio, impactando la calidad de vida y las actividades de la vida diaria de los pacientes ${ }^{6}$. Por otra parte, los beneficios del ejercicio se relacionan con el aumento del consumo de oxígeno máximo $\left(\mathrm{VO}_{2 \mathrm{max}}\right)$, la capacidad física, la función del músculo esquelético, la mejor función endotelial, el incremento del tono vagal y la disminución del tono simpático cuando se realizan en programas de rehabilitación cardiaca de mayor de duración ${ }^{7}$. Sin embargo, los cambios en la capacidad aeróbica se han descrito en pocas ocasiones en programas de menor duración, por lo que este estudio tiene como objetivo determinar cuáles son los cambios en la capacidad física y algunas variables fisiológicas en un grupo de pacientes con insuficiencia cardiaca al realizar 6 y 12 semanas de un programa de rehabilitación cardiaca en una clínica de la ciudad de Cali, en Colombia.

\section{Método}

Se realizó un estudio de tipo descriptivo longitudinal, en los meses de septiembre a diciembre de 2017, en pacientes con diagnóstico de insuficiencia cardiaca que ingresaron al programa de rehabilitación cardiaca de una clínica de cuarto nivel de la ciudad de Cali, 
Colombia, y a los cuales se les realizaron mediciones antropométricas y una prueba de esfuerzo incremental tipo Bruce modificado en los tres momentos de rehabilitación (inicio y semanas 6 y 12) ${ }^{8}$.

\section{Selección de los pacientes}

Mediante un muestreo no probabilístico se incluyeron por convencinecia todos los pacientes con insuficiencia cardiaca que ingresaron al programa de rehabilitación cardiaca de la clínica, siendo un total de 23, los cuales previamente fueron diagnosticados con insuficiencia cardiaca por un médico especialista en cardiología mediante ecocardiografía transtorácica realizada al menos 2 meses antes de vincularse al estudio. Los pacientes estaban estratificados en estadio $C$ y clase funcional II-III de la clasificación de la New York Heart Association $(\mathrm{NYHA})^{9}$. Fueron excluidos del estudio los pacientes con clase funcional NYHA IV y los que tuvieran alguna limitación para realizar movimientos activos y resistidos (fracturas recientes, alteraciones hemodinámicas recientes, evento de enfermedad coronaria posterior al diagnóstico de insuficiencia cardiaca, enfermedades infecciosas, limitación neuromuscular).

El comité de investigación y de ética humana de la Clínica aprobó el estudio, y de todos los pacientes se obtuvo el consentimiento informado por escrito.

\section{Mediciones}

Se registraron en todos los pacientes las características sociodemográficas, tales como sexo y edad. Al inicio y al final del programa de rehabilitación cardiaca se midieron las condiciones antropométricas, como la talla con un tallímetro Krammer ${ }^{\circledR}$ (Holtain Ltd., Crymych Dyfed, R.U.) de cuatro segmentos y $1 \mathrm{~mm}$ de precisión, y el peso medido con una balanza de piso Health-o-Meter $^{\circledR}$ (Continental Scale Corp., Bridgeview, III, EE.UU.) con $100 \mathrm{~g}$ de precisión; a su vez, se determinó el índice de masa corporal (IMC) en $\mathrm{kg} / \mathrm{m}^{2}$. Se tomaron parámetros fisiológicos, como la frecuencia cardiaca en reposo y al final de la prueba de ejercicio. La presión arterial sistólica (PAS) y diastólica (PAD) en reposo y al final de la prueba de ejercicio se midieron con tensiómetro y esfingomanómetro aneroide (WelchAllyn ${ }^{\circledR}$ DS44$11 C B T$ ) previamente calibrado, y sus resultados se presentaron en $\mathrm{mmHg}$. Finalmente, la prueba de esfuerzo se realizó adoptando las indicaciones del American College of Cardiology y la American Heart Association?. El protocolo utilizado por los pacientes fue el Bruce modificado en banda $\sin$ fin $^{10}$. Se estimó el $\mathrm{VO}_{2} \max$ mediante la ecuación: $\mathrm{VO}_{2} \max =3.5+0.1$ (velocidad) +1.8 (velocidad) (grado), y MET $=\mathrm{VO}_{2} \mathrm{max} / 3.5^{11}$.

\section{Programa de ejercicio físico en rehabilitación cardiaca}

En la primera sesión se determinó la estratificación del riesgo según las recomendaciones de la American Heart Association ${ }^{12}$. Se realizó examen osteomuscular (funcional), toma de signos vitales, peso, talla e IMC, y se realizó la prueba de esfuerzo con el fin de determinar la capacidad aeróbica de los pacientes.

El programa de rehabilitación cardiaca consistió en ejercicio durante tres sesiones por semana y 12 semanas de duración, realizando 60 minutos por sesión. Los pacientes realizaron calentamiento de 5 minutos con actividades de autocarga y posiciones (bípedo-sedente), entrenamiento de fuerza por 20 minutos con un peso mínimo de 2 libras y un peso máximo de 6 libras, aumentando 2 libras cada 4 semanas, realizando tres o cuatro series de 12 a 15 repeticiones; la intensidad del ejercicio se trabajó al 50\% de la frecuencia cardiaca máxima de reserva por Karvonnen ${ }^{13}$ en prueba de esfuerzo, y se incrementó hasta el $70 \%$ de la frecuencia cardiaca máxima al final del entrenamiento ${ }^{14,15}$. Mediante la escala de percepción del esfuerzo de 0 a 10 de Borg modificada se monitorizaron aquellos pacientes que no alcanzaran la intensidad máxima de frecuencia cardiaca por el uso de betabloqueadores ${ }^{16}$.

\section{Análisis estadístico}

La información recolectada fue anexada en una base de datos en Excel y posteriormente se exportó al programa SPSS versión 21, donde además se interpretaron los datos. Se realizó inicalmente una descripción de los datos presentando las variables cualitativas como frecuencia y porcentaje; para las variables cuantitativas se utilizó la prueba de Shapiro-Wilk y se determinó el comportamiento paramétrico de las variables resultado presentándolas como media y desviación estándar. Utilizando la prueba de medidas repetidas, se tuvo en cuenta la homogeneidad entre las varianzas y el cumplimiento de la esfericidad de cada una de las variables en los tres momentos (inicio, 6 y 12 semanas). La significación estadística fue de un 0.05 entre las variables.

\section{Resultados}

En la tabla 1 se describen los resultados de la caracterización sociodemográfica de los pacientes, en la 
Tabla 1. Características sociodemográficas de los pacientes $(n=23)$

\begin{tabular}{|l|c|}
\hline Variables & Frecuencia (\%) \\
\hline Edad (media \pm DE) & $59.09 \pm 2.27$ \\
\hline Nivel de escolaridad, $\mathrm{n}(\%)$ & \\
\hline Primaria completa & $10(43.5)$ \\
Bachillerato completo & $4(17.4)$ \\
Técnico & $4(17.4)$ \\
\hline Profesional & $5(21.7)$ \\
\hline Sexo, $\mathrm{n}(\%)$ & \\
\hline Hombre & $18(78.3)$ \\
\hline Mujer & $5(21.7)$ \\
\hline Estado civil, $\mathrm{n}(\%)$ & \\
\hline Soltero & $1(4.3)$ \\
\hline Casado & $11(47.8)$ \\
\hline Separado & $5(21.7)$ \\
Unión libre & $4(17.4)$ \\
\hline Viudo & $2(8.7)$ \\
\hline Lugar de residencia, $\mathrm{n}(\%)$ & \\
\hline Urbano & $20(87.0)$ \\
\hline Rural & $3(13.0)$ \\
\hline Empresa promotora de salud, $\mathrm{n}(\%)$ & \\
\hline Contributivo & $17(74.0)$ \\
\hline Subsidiado & $6(26.0)$ \\
\hline Estrato socioeconómico, $\mathrm{n}(\%)$ & \\
\hline Bajo 1-2 & $5(21.7)$ \\
\hline Medio 3-4 & $15(65.2)$ \\
\hline Alto 5-6 & $3(13.0)$ \\
\hline
\end{tabular}

DE: desviación estándar.

cual se evidencia que el sexo de mayor participación fueron el masculino, con una relación de cuatro de cada cinco individuos. Los niveles de escolaridad más frecuentes que tenían los participantes fueron primaria completa, en el $43 \%(n=10)$, seguido del nivel profesional con el $21 \%(n=5)$. Respecto al estado civil, uno de cada dos participantes era casado. Para el lugar de residencia, la población mostró una distribución concentrada en la zona urbana, ya que el $87 \%(n=20)$ residía en esta zona. El régimen más frecuente al cual se encontraban afiliados los participantes fue el contributivo, ya que tres de cada cuatro individuos correspondían a este. Finalmente, el estrato social que predominó en la población fue el estrato medio, con un $65 \%(n=15)$. El promedio de la fracción de eyección del ventrículo izquierdo (FEVI) de los pacientes fue de $34.9 \pm 1.4$. En cuanto a los cambios luego de la rehabilitación cardiaca, en la tabla 2 se presentan los observados en las semanas 6 y 12 de intervención.

El cambio de los valores absolutos de las variables peso e IMC fue mínimo durante las 12 semanas de rehabilitación; este cambio no mostró diferencia estadísticamente significativa ( $p>0.05)$. Las mediciones de la PAS inicial y final mostraron comportamientos similares, y se evidenció una disminución al final de la rehabilitación de $12.2 \mathrm{mmHg}$ para la PAS inicial y de $17.4 \mathrm{mmHg}$ para la PAS final; estas disminuciones mostraron diferencias estadísticamente significativas $(p<0.05)$ entre los valores de la semana 1 y de la semana 6, tiempo en el cual se registró el mayor cambio: $10 \mathrm{mmHg}$ para la PAS inicial y $17.4 \mathrm{mmHg}$ para la PAS final. La PAD inicial y final también tuvo un comportamiento similar, disminuyendo su valor hasta la semana 6 , pero el valor final en la semana 12 fue superior a la medición de la semana 6 .

Se evidenció que la frecuencia cardiaca inicial disminuyó $6.2 \mathrm{l} / \mathrm{min}$ al final de la rehabilitación, pero el mayor cambio se registró durante las primeras 6 semanas, con una reducción de $6.8 \mathrm{l} / \mathrm{min}$, siendo esta última estadísticamente significativa $(p=0.005)$. Por el contrario, la frecuencia cardiaca final mostró un incremento progresivo durante las fases de rehabilitación, pero no se encontraron diferencias estadísticamente significativas.

Finalmente, las variables de consumo de oxígeno y MET mostraron un incremento progresivo durante la rehabilitación, aumentando $3.6 \mathrm{ml} / \mathrm{kg} / \mathrm{min}$ y $1.1 \mathrm{ml} / \mathrm{kg} / \mathrm{min}$, respectivamente, con diferencias estadísticamente significativas en los tres momentos, pero se evidenció un mayor cambio entre las semanas 1 y 6 , altamente significativo $(p=0.00)$, donde se registró un cambio de $2.2 \mathrm{ml} / \mathrm{kg} / \mathrm{min}$ para la variable consumo de oxígeno y de $0.6 \mathrm{ml} / \mathrm{kg} / \mathrm{min}$ para la variable MET.

\section{Discusión}

La insuficiencia cardiaca afecta a 26 millones de personas en el mundo y actualmente se considera como un importante problema de salud pública ${ }^{17}$. En los países desarrollados, su prevalencia es aproximadamente del $1-2 \%$ en la población adulta y llega al 10\% en personas mayores de 70 años $^{2}$. En Latinoamérica, los reportes muestran una prevalencia del $2.3 \%$ con tendencia a aumentar por el envejecimiento de la población y el incremento de los factores de riesgo cardiovascular ${ }^{18}$; esto evidencia que la insuficiencia cardiaca es un problema de salud pública que amerita intervenciones basadas en las necesidades de los pacientes.

El tratamiento de la insuficiencia cardiaca está compuesto por dos ejes principales: el tratamiento farmacológico $^{19}$ y el tratamiento no farmacológico, como la 
Rev Colomb Cardiol. 2021;28(5)

Tabla 2. Cambios durante la rehabilitación cardiaca $(n=23)$

\begin{tabular}{|c|c|c|c|c|}
\hline Variables & Inicio & 6 semanas & 12 semanas & $\mathbf{p}$ \\
\hline Peso, kg & $73.0 \pm 12.9$ & $73.0 \pm 12.7$ & $720.7 \pm 12.6$ & 0.88 \\
\hline \multirow[t]{2}{*}{ Índice de masa corporal, kg/m² } & $26.5 \pm 4.3$ & $26.6 \pm 4.4$ & $26.5 \pm 4.6$ & \multirow[t]{2}{*}{0.90} \\
\hline & & $=0.8 P=0.5$ & & \\
\hline \multirow[t]{2}{*}{ Presión arterial sistólica inicial, mmHg } & $121.3 \pm 11.8$ & $111.3 \pm 14.6$ & $109.1 \pm 14.4$ & \multirow[t]{2}{*}{0.015} \\
\hline & & $=0.012 \mathrm{P}=0$. & & \\
\hline \multirow[t]{2}{*}{ Presión arterial diastólica inicial, mmHg } & $76.9 \pm 8.2$ & $68.7 \pm 7.7$ & $70.43 \pm 8.2$ & \multirow[t]{2}{*}{0.001} \\
\hline & & $=0.000 \mathrm{P}=0$ & & \\
\hline \multirow[t]{2}{*}{ Presión arterial sistólica final, $\mathrm{mmHg}$} & $120 \pm 15.1$ & $105.7 \pm 15.6$ & $102.6 \pm 13.9$ & \multirow[t]{2}{*}{0.001} \\
\hline & & $=0.000 \mathrm{P}=\mathrm{C}$ & & \\
\hline \multirow[t]{2}{*}{ Presión arterial diastólica final, $\mathrm{mmHg}$} & $73.3 \pm 8.2$ & $67.4 \pm 7.5$ & $68.7 \pm 8.2$ & \multirow[t]{2}{*}{0.037} \\
\hline & & $=0.014 \mathrm{P}=\mathrm{C}$ & & \\
\hline \multirow[t]{2}{*}{ Frecuencia cardiaca inicial, lat/min } & $79 \pm 13.6$ & $72.2 \pm 10.1$ & $72.8 \pm 9.6$ & \multirow[t]{2}{*}{0.015} \\
\hline & & $=0.005 \mathrm{P}=0$ & & \\
\hline \multirow[t]{2}{*}{ Frecuencia cardiaca final, lat/min } & $105.5 \pm 20.7$ & $108.4 \pm 17.8$ & $111.9 \pm 13.7$ & \multirow[t]{2}{*}{0.40} \\
\hline & & $P=0.3 P=0$. & & \\
\hline \multirow[t]{2}{*}{ Consumo de oxígeno, $\mathrm{ml} / \mathrm{kg} / \mathrm{min}$} & $16.1 \pm 1.9$ & $18.3 \pm 2.8$ & $19.7 \pm 3.5$ & \multirow[t]{2}{*}{0.000} \\
\hline & & $=0.000 \mathrm{P}=0$ & & \\
\hline \multirow[t]{2}{*}{ Índice metabólico basal, MET } & $4.6 \pm 0.6$ & $5.2 \pm 0.8$ & $5.7 \pm 1$ & \multirow[t]{2}{*}{0.000} \\
\hline & & $=0.000 \mathrm{P}=0$ & & \\
\hline
\end{tabular}

rehabilitación cardiaca, en la que se enfatiza el ejercicio físico, en especial el aeróbico, reconociendo que proporciona importantes beneficios al mejorar la calidad de vida relacionada con la salud y reducir las hospitalizaciones relacionadas con la insuficiencia cardiaca. Por otra parte, se ha descrito que los beneficios del ejercicio físico son independientes de la edad, el sexo, el grado de disfunción del ventrículo izquierdo y el entorno de los programas de ejercicios ${ }^{20}$. Es claro que el ejercicio aeróbico durante 3 meses en los paciente con insuficiencia cardiaca mejora el $\mathrm{VO}_{2}$ y la capacidad física ${ }^{21}$, lo que claramente se evidencia en este estudio, pero aún no se han documentado con suficiente rigurosidad los cambios que pueden producirse en el $\mathrm{VO}_{2}$ y otras variables fisiológicas en los pacientes que no culminan el programa de rehabilitación cardiaca o que realizan programas de menor duración.

Los pacientes de este estudio tenían un promedio de edad de $59.09 \pm 2.27$ años, que está por debajo de la edad de presentación reportada en Colombia, donde la mayor incidencia de la enfermedad se da en personas entre los 65 y los 94 años, lo que puede obdecer a que este grupo de paccientes son categorizados con mayor compromiso funcional dadas las características de inclusión del estudio según la escala NYHA. Además, el sexo más afectado por esta enfermedad en Colombia es el masculino, lo que coincide con este estudio, ya que el $80 \%$ de los participantes eran hombres $^{22}$. Por otra parte, se evidenció que el $50 \%$ de los pacientes eran casados, el $87 \%$ tenían como lugar de residencia la zona urbana, el $75 \%$ pertenecían al régimen contributivo y el $65 \%$ eran del estrato social medio, lo cual contrasta con otra investigación desarrollada en la ciudad de Cali, en la cual la mayor parte de los participantes pertenecían a la zona rural y al estrato social bajo ${ }^{23}$. Esto sugiere que la población con estrato social más alto tiene mayores posibilidades de acceso a los programas de rehabilitación cardiaca, ya sea porque residen en la ciudad donde se encuentra 
ubicada la clínica o porque tienen mayores recursos para realizar pagos por desplazamientos los 3 días a la semana por 12 semanas.

Algunos investigadores, como Ramírez-Araya, et al. ${ }^{24}$ en Costa Rica, han reportado incrementos del $\mathrm{VO}_{2}$ que van desde un $10 \%$ a un $31 \%$ después de asistir por diferentes periodos de tiempo a un programa de rehabilitación. Un estudio ${ }^{23}$ mencionó cambios positivos a las 6 semanas en el $\mathrm{VO}_{2}$ (hombres $1,3 \pm 2$ vs. mujeres $0.7 \pm 1$ ) en una muestra de 15 pacientes, medidos por medio del test de la caminata de 6 minutos. Aunque nuestra medición no se realizó con el test de la caminata de 6 minutos, debido a que se realizó una prueba de esfuerzo, los datos concuerdan con la presente investigación, en la que el cambio en el $\mathrm{VO}_{2}$ de los pacientes fue altamente significativo $(p=0.000)$ a las 6 semanas de rehabilitación. Esto podría explicarse por las adaptaciones iniciales producidas por el ejercicio aeróbico continuo, sumado al fortalecimiento muscular, en el cual, entre otras cosas, se produce un aumento de la masa muscular y de la tasa metabólica basal, y por consiguiente aumenta el gasto energético ${ }^{25}$, posibilitando una mayor eficiencia muscular y un mejor desempeño en pruebas de ejercicio.

Nuestra investigación mostró cambios estadísticamente significativos en el $\mathrm{VO}_{2}$ en los tres momentos de medición: de $16.1 \pm 1.9$ en la primera medición a 18.3 \pm 2.8 en la semana 6 y a $19.7 \pm 3.5$ en la semana 12 . Esto difiere de la investigación realizada por Lugo, et al. ${ }^{26}$, que tuvo como objetivo evaluar el efecto de un programa supervisado sobre el $\mathrm{VO}_{2}$ y la calidad de vida en pacientes con insuficiencia cardiaca, en un ensayo clínico con doble enmascaramiento en el cual se asignaron grupos paralelos (23 en el grupo de intervención y 26 en el grupo control) y se realizaron mediciones del $\mathrm{VO}_{2}$ a las 8 semanas y a los 6 meses con una prueba de esfuerzo, que no mostró cambios estadísticamente significativos en ninguno de los momentos de medición. No obstante, los autores reportan que una de las limitaciones de su estudio fue la adherencia al tratamiento, del 78\%; situación que no sucedió en nuestro estudio, posiblemente porque el entrenamiento en este estudio comprendía ejercicio de predominio aeróbico y fortalecimiento muscular. También es importante tener en cuenta que, en cuanto al sexo, los participantes de este estudio eran en su mayoría hombres, lo que claramente se relaciona con la adherencia y el desempeño en el ejercicio, evidenciando mayores mejorías en las primeras 6 semanas con una diferencia de medias de 2.2, comparado con 1.4 para las 12 semanas en el $\mathrm{VO}_{2}$. Estos cambios durante las primeras 6 semanas pueden explicarse porque en las adaptaciones inmediatas se presentan debido a la mayor disponibilidad de sistemas energéticos, el mejor acoplamiento de las miofibrillas para el desempeño muscular y la supercompensación, reconocidas como manifestaciones agudas al ejercicio; en cambio, las adaptaciones a mediano y corto plazo se expresan con un menor incremento de la capacidad aeróbica, pero con la capacidad de mantener estas adaptaciones por más tiempo ${ }^{26}$.

Unos valores altos de $\mathrm{VO}_{2}$ han demostrado un mejoramiento directo sobre el sistema nervioso autónomo, con adaptaciones positivas en la respuesta cronotrópica y la recuperación de la frecuencia cardiaca después del ejercicio ${ }^{27}$. Tonguino, et al. ${ }^{23}$ no encontraron en su investigación diferencias estadísticamente significativas en la variación de la frecuencia cardiaca a la semana 6 de intervención, lo que se relaciona con nuestro estudio, ya que en solo una de las cuatro mediciones de la frecuencia cardiaca se observó una disminución estadísticamente significativa, y esta fue la frecuencia cardiaca inicial a las 6 semanas de rehabilitación, lo que seguramente se atribuye a la naturaleza de los pacientes y su tratamiento, que incluye el uso de betabloqueadores.

Respecto a los MET obtenidos en las tres mediciones, en 2010 se desarrolló una investigación con una muestra de 214 pacientes en la que se pretendía establecer el impacto de la rehabilitación cardiaca en pacientes con insuficiencia cardiaca de origen isquémico con fracción de eyección menor del $40 \%$, utilizando la percepción subjetiva por medio de la escala de Borg, el $\mathrm{VO}_{2}$, los MET y la distancia en millas, antes y al finalizar la terapia de rehabilitación cardiovascular. Se encontró que los pacientes mejoraron en MET, pasando de un promedio de 2,22 $\pm 0,6$ a 5,44 $\pm 1,9$; además, se identificó que las personas que asistieron a más de 18 sesiones de rehabilitación incrementaron más este valor que aquellas que asistieron a menos de 18 sesiones, con una diferencia estadísticamente significativa $(p<0.02)^{28}$. Estos resultados son similares a los encontrados en nuestra investigación, ya que el promedio de fracción eyección de los pacientes fue de $34.9 \pm 1.4$, lo que corresponde a una similitud clínica de los pacientes, y además hubo un incremento en los MET, cuya primera medición tuvo como promedio 4.6 \pm 0.6 , a las 6 semanas $5.2 \pm 0.8$ y a las 12 semanas $5.7 \pm 1$. Estos hallazgos incrementales en los MET en nuestro estudio también obedecen a una adecuada prescripción del ejercicio, tomando la frecuencia cardiaca máxima en la prueba de esfuerzo y utilizando el método de Karvonen para prescribir ejercicio. 
Los programas de rehabilitación cardiaca que incluyen ejercicio físico y soporte médico y psicológico logran disminuir la mortalidad entre un $20 \%$ y un $30 \%$, mejoran la capacidad funcional entre un $10 \%$ y un $34 \%$, y adicionalmente logran descensos en la presión arterial y mejoras en la calidad de vida ${ }^{24}$. Lo mencionado anteriormente se relaciona con lo encontrado en nuestra investigación, en la que se halló una disminución estadísticamente significativa de la PAS y la PAD inicial y final hasta la semana 6 de rehabilitación. Estos resultados se pueden comparar con los obtenidos en 2012 por Pérez-Aldama, et al. ${ }^{29}$ en la Habana, Cuba, donde realizaron un estudio prospectivo, observacional y analítico para evaluar la eficacia de un programa de rehabilitación cardiovascular en pacientes con insuficiencia cardiaca crónica de etiología isquémica, y encontraron que hubo una disminución estadísticamente significativa en la PAS y la PAD después de 6 meses de rehabilitación, lo que demuestra que la continuación en el tiempo de intervención en los programas de rehabilitación cardiaca favorece el mejoramiento de algunas variables fisiológicas, debido a la disminución en las resistencias arteriales periféricas, las cuales presentan una mayor dilatación vasoarterial favoreciendo la disminución de la presión arterial en los pacientes con insuficiencia cardiaca ${ }^{30}$.

Finalmente, en las variables de peso e IMC no se observaron cambios significativos ( $p>0.05$ ). Estos resultados son similares a los obtenidos por Pérez-Aldama, et al..$^{29}$, quienes luego de realizar un entrenamiento a pacientes con insuficiencia cardiaca por 12 meses de rehabilitación cardiaca no encontraron modificaciones en el IMC. Sin embargo, resultados contrarios fueron descritos por Yuriko T, et al..$^{31}$ en Japón tras un programa de rehabilitación cardiaca de aproximadamente 5 meses y una frecuencia de cinco veces por semana, donde se evidenció una disminución del peso corporal y de la masa grasa estadísticamente significativa $(p<0.05)$, lo que sugiere que el ejercicio no es la única variable que influye sobre las modificaciones del peso corporal en este tipo de pacientes, y resulta sumamente importante que los programas de rehabilitación cardiaca promuevan cambios en los hábitos alimenticios.

Las limitaciones principales de este estudio radican en que no se pudo obtener la vinculación de la población mediante un muestreo, ya que se vincularon por conveniencia, por lo cual no se pueden extrapolar resultados ni generalizar a la población con insuficiencia cardiaca en general. Por otra parte, en futuras investigaciones es necesario integrar la multidisciplinariedad de los programas de rehabilitación cardiaca, lo que permitirá controlar algunos factores de riesgo y posibilitar cambios en otras variables, tales como datos antropométricos y ansiedad/depresión de los pacientes. Es importante tener en cuenta que este estudio no contempló realizar la medición final de la FEVI para establecer cambios estructurales asociados al ejercicio físico.

La principal fortaleza de este estudio es el análisis de las variables fisiológicas en diferentes momentos durante la rehabilitación cardiaca, lo que favorece la comprensión de las adaptaciones de pacientes que realizan ejercicio según el número de semanas de entrenamiento. Por otra parte, el protocolo de intervención es una estrategia favorable para los pacientes vinculados al programa, ya que mediante la prueba de esfuerzo se obtuvieron datos confiables para la adecuada prescripción de ejercicio en esta población.

\section{Conclusiones}

La caracterización sociodemográfica de los pacientes tuvo semejanzas con los datos epidemiológicos reportados para Colombia. La única variable fisiológica que no mostró un cambio significativo durante las primeras 6 semanas de rehabilitación fue la frecuencia cardiaca final; el $\mathrm{VO}_{2}$ y el índice metabólico basal tuvieron un incremento progresivo a lo largo de las 12 semanas de rehabilitación cardiaca, observándose su mayor incremento en las primeras 6 semanas.

\section{Financiamiento}

Los autores declaran no haber recibido financiamiento.

\section{Conflicto de intereses}

Ninguno de los autores manifiesta tener conflictos de intereses en el presente estudio.

\section{Agradecimientos}

Los autores agradecen profundamente a la Clínica de Occidente S.A. y a los pacientes que voluntariamente participaron en el estudio.

\section{Responsabilidades éticas}

Protección de personas y animales. Los autores declaran que los procedimientos seguidos se conformaron a las normas éticas del comité de experimentación 
humana responsable y de acuerdo con la Asociación Médica Mundial y la Declaración de Helsinki.

Confidencialidad de los datos. Los autores declaran que han seguido los protocolos de su centro de trabajo sobre la publicación de datos de pacientes.

Derecho a la privacidad y consentimiento informado. Los autores han obtenido el consentimiento informado de los pacientes y/o sujetos referidos en el artículo. Este documento obra en poder del autor de correspondencia.

\section{Bibliografía}

1. Organización Mundial de la Salud. 2017. (Acceso 3 de marzo de 2018.) Disponible en: http://www.who.int/mediacentre/factsheets/fs355/es/.

2. Ponikowski P, Anker S, Voors A. 2016 ESC Guidelines for the diagnosis and treatment of acute and chronic heart failure. Eur $\mathrm{J}$ Heart Fail. 2016;18:891-975

3. Pons F, Lupon A, Urrutia A. Mortalidad y causas de muerte en pacientes con insuficiencia cardiaca: experiencia de una unidad especializada multidisciplinaria|. Rev Esp Cardiol. 2010;63:303-14.

4. Pereira-Rodríguez J, Rincón González G, Niño Serrato D. Insuficiencia cardíaca: aspectos básicos de una epidemia en aumento. CorSalud. 2016;8:58-70.

5. McMurray JJ, Adamopoulos S, Anker SD, Auricchio A, Böhm M, et al. ESC Guidelines for the diagnosis and treatment of acute and chronic heart failure 2012: The Task Force for the Diagnosis and Treatment of Acute and Chronic Heart Failure 2012 of the European Society of Cardiology. Developed in collaboration with the Heart Failure Association (HFA) of the ESC. Eur J Heart Fail. 2012;14:803-69.

6. Taylor R, Sagar V, Davies E, Briscoe S, Coats AJ, Dalal H, et al. Exercisenbased rehabilitation for heart failure. Cochrane Database Syst Rev. 2014;(4):CD003331.

7. Piepoli M, Davos C, Francis D. Exercise training meta-analysis of trials in patients with chronic heart failure (ExTraMATCH). BMJ. 2004;328:189.

8. Gibbons RJ, Balady GJ, Beasley JW, Bricker JT, Duvernoy WF, Froelicher VF, et al. ACC/AHA Guidelines for Exercise Testing. A report of the American College of Cardiology/American Heart Association Task Force on Practice Guidelines (Committee on Exercise Testing). J Am Coll Cardiol. 1997;30:260-311.

9. Hurts W, Morris D, Alexander W. The use of the New York Heart Association's classification of cardiovascular disease as part of the patient's complete problem list. Clin Cardiol. 1999;22:385-90.

10. Arós F, Boraita A, Alegría E, Alonso AM, Bardají A, Lamiel R, et al. Guías de práctica clínica de la Sociedad Española de Cardiología en pruebas de esfuerzo. Rev Esp Cardiol. 2000;53:1063-94.

11. Pescatello L, Riebe D, Arena R, Thompson P. ACSM's Guidelines for exercise testing and prescription. Lippincott Williams \& Wilkins 9a. Edición, Philadelphia, EUA, 2014.

12. Navas C, Lugo L, Ortiz S. Estudio descriptivo del programa de rehabilitación cardiaca de la Clínica Las Américas. Rev Col Cardiol. 2011;18: 199-205.

13. Karvonen $\mathrm{MJ}$, Kentala $\mathrm{E}$, Mustala $\mathrm{O}$. The effects of training on heart rate; a longitudinal study. Ann Med Exp Biol Fenn. 1957;35307-15.
14. Ávila-Valencia JC, Hurtado-Gutiérrez H, Benavides-Córdoba V, Betancourt-Peña J. Ejercicio aeróbico en pacientes con falla cardiaca con y sin disfunción ventricular en un programa de rehabilitación cardiaca. Rev Col Cardiol. 2019;26:162-8.

15. Ávila JC, Betancourt-Peña J. Cambios en el perfil lípidico y algunas variables antropométricas en pacientes con enfermedad coronaria que culminaron un programa de rehabilitación cardiaca. Rev MC. 2014;8:18-25.

16. Atheortua D, Gallo J, Rico M, Durango L. Efecto de un programa de rehabilitación cardiaca basado en ejercicio sobre la capacidad física, la función cardiaca y la calidad de vida, en pacientes con falla cardiaca. Rev Col Cardiol. 2011;18:25-36.

17. Yancy CW, Jessup M, Bozkurt B, Butler J, Casey DE Jr, Drazner MH, et al. 2013 ACCF/AHA guideline for the management of heart failure: a report of the American College of Cardiology Foundation/American Heart Association Task Force on Practice Guidelines. J Am Coll Cardiol. 2013;62:e147-239.

18. Saldarriaga Cl. Editorial suplemento clínicas de falla cardiaca. Rev Col Cardiol. 2016;23(Supl 1).

19. Jaramillo C, Gómez E, Hernández E, Saldarriaga C, Flórez N, Buitrago R. Consenso colombiano para el diagnóstico y tratamiento de la insuficiencia cardiaca crónica. Sociedad Colombiana de Cardiología y Cirugía Cardiovascular. 2015. Disponible en: https://scc.org.co/wp-content/ uploads/2012/2015/01/agudawebcompleta.pdf

20. Sagar VA, Davies EJ, Briscoe S, Coats AJ, Dalal HM, Lough F, et al. Exercise-based rehabilitation for heart failure: systematic review and meta-analysis. Open Heart. 2015;2:e000163.

21. Smart N. How do cardiorespiratory fitness improvements vary with physical training modality in heart failure patients? A quantitative guide. Exp Clin Cardiol. 2013;18:e21.

22. Gómez E. Capítulo 2. Introducción, epidemiología de la falla cardiaca e historia de las clínicas de falla cardiaca en Colombia. Rev Col Cardiol. 2016;23:6-12.

23. Tonguino-Rosero S, Wilches-Luna EC, Escobar-Durán N, Castillo-Vergara JJ. Impacto de 6 semanas de rehabilitación cardiaca en la capacidad funcional y en la calidad de vida de pacientes con enfermedades cardiovasculares. Rehabilitacion. 2014;48:204-9.

24. Ramírez-Araya F, Ureña Bonilla P, Romero Blanco L. Efecto de un programa de ejercicios en la capacidad funcional y la respuesta hemodinámica de pacientes con enfermedad cardiovascular. Rev Costarric Cardiol. 2016;16:5-11.

25. Jorquera C, Cancino J. Ejercicio, obesidad y síndrome metabólico. Rev Med Clin Las Condes. 2012;23:227-35.

26. Lugo LH, Navas C, Plata J. Ensayo clínico aleatorizado para evaluar el efecto de un programa de rehabilitación cardiaca supervisado con ejercicio en el consumo de oxígeno, la función y la calidad de vida en pacientes con falla cardiaca crónica. Rev Col Cardiol. 2017;25:106-15.

27. Lazzeroni D, Castiglioni P, Bini M. Improvement in aerobic capacity during cardiac rehabilitation in coronary artery disease patients: is there a role for autonomic adaptations? Eur J Prev Cardiol. 2016;24:357-64.

28. Quiroz C, Sarmiento J, Jaramillo C. Impacto de la rehabilitación cardiaca en pacientes con falla cardiaca de origen isquémico. Rev Col Cardiol. 2010;18:10-24.

29. Pérez Aldama L, Rodríguez Cuba A, Llanes K. Rehabilitación cardiovascular en pacientes con insuficiencia cardiaca crónica de etiología isquémica. Rev Cuba Investig Biomed. 2012;31:437-46.

30. Pearson M, Smart N. Aerobic training intensity for improved endothelial function in heart failure patients: a systematic review and meta-analysis. Cardiol Res Pract. 2017;2017:2450202.

31. Yuriko T, Shusuke Y, Takayuki I. Improved exercise capacity after cardiac rehabilitation is associated with reduced visceral fat in patients with chronic heart failure. Int Heart J. 2017;58:746-51. 